\title{
Semantic Lexicon Adaptation for Use in Query Interpretation
}

\author{
Ana-Maria Popescu \\ Yahoo! Labs \\ Sunnyvale, CA \\ 94089 \\ amp@yahoo-inc.com
}

\author{
Patrick Pantel \\ Yahoo! Labs \\ Sunnyvale, CA \\ 94089 \\ ppantel@yahoo-inc.com
}

\author{
Gilad Mishne \\ Yahoo! Labs \\ Sunnyvale, CA \\ 94089 \\ gilad@yahoo-inc.com
}

\begin{abstract}
We describe improvements to the use of semantic lexicons by a state-of-the-art query interpretation system powering a major search engine. We successfully compute concept label importance information for lexicon strings; lexicon augmentation with such information leads to a $6.4 \%$ precision increase on affected queries with no query coverage loss. Finally, lexicon filtering based on label importance leads to a $13 \%$ precision increase, but at the expense of query coverage.
\end{abstract}

\section{Categories and Subject Descriptors}

H.3.3 [Information Systems]: Information Storage and Retrieval

\section{General Terms}

Algorithms, Experimentation, Measurement

\section{INTRODUCTION}

An important aspect of query interpretation is recognizing the types of entities mentioned in a user query. We focus on a particular aspect of entity tagging in queries: the use of semantic domain knowledge in the form of semantic lexicons, whose limitations can include limited coverage, noise and finally, ambiguity of lexicon entries, which is the focus of this poster. A high-precision, large semantic lexicon extracted from Web data may contain few ontological errors, but many ambiguous strings - some with a different primary sense: e.g., given an Actor lexicon, clint eastwood and mel gibson are strings for which Actor is an important sense, while snoop dogg is not. Our work computes label importance information for lexicon strings in order to enrich the semantic lexicons supplied to a client query interpretation system for better performance.

Web Query Interpretation with QIS

QIS is a state-of-the-art query interpretation engine which powers a major search engine. Given a query, QIS produces the most likely interpretations for it: an interpretation consists of a set of a semantic types assigned to non-overlapping query tokens. QIS makes use of a large type taxonomy and uses a combination of taggers (both statistical and semantic lexicon lookups) in order to generate candidate interpretations of the query. Next, a ranking module assigns a score

Copyright is held by the author/owner(s).

WWW 2010, April 26-30, 2010, Raleigh, North Carolina, USA.

ACM 978-1-60558-799-8/10/04.
Table 1: Semantic Lexicon Entries: Examples

\begin{tabular}{|l|l|l|}
\hline $\begin{array}{l}\text { Actor }= \\
\text { Important label }\end{array}$ & $\begin{array}{l}\text { Actor }= \\
\text { Minor label }\end{array}$ & $\begin{array}{l}\text { Actor }= \\
\text { Incorrect label }\end{array}$ \\
\hline al pacino & karen mulder & girls \\
robert de niro & sharon osborne & han solo \\
robert redford & snoop dogg & mark twain \\
tom hanks & martin scorsese & hollywood \\
\hline \hline
\end{tabular}

to each interpretation; ranking is approached as a supervised learning task, where manual judgments of the quality of interpretations for randomly-selected queries are used as training data. A multitude of features is used to represent each interpretation, including both query-level features (e.g., number of words, the name of taggers which participated in forming the interpretation) and tag-level features (e.g., tagger confidence).

\section{CONCEPT LABEL IMPORTANCE}

We describe an annotation task which estimates concept label importance for strings in a lexicon and is used for gold standard data set construction.

Importance Annotation Consider the string $s=$ al pacino in the Actor lexicon. We retrieve the queries containing $s$ and rank them by their frequency; we retain the top $k$ queries whose cumulative frequency is more than $80 \%$ of the total frequency of queries containing $s$. For each query $q$, we manually examine the top 10 web search results and record the \% of pages $r$ in which al pacino's Actor sense appears dominant. The score for (Actor, $s$ ) is the sum of $r_{1}, \ldots r_{k}$, each weighted by the relative query frequency for $q_{i}$ : e.g., the scores for the Actor label and al pacino, newman are 1 and, respectively, 0.78 . We use 0.2 as a manually chosen importance threshold on score values.

We detect label importance using two main methods:

M1. $I_{C M}$ uses a supervised $M L$ approach (Gradient Boosted Decision Trees [1]). The training set is a sample of the target lexicon manually labeled as described above. Features include generic string-level ambiguity features and features for concepts in the QIS taxonomy (see Table 2).

M2. Given a set of concepts and a string $s, I_{M M}$ computes an estimate of $P\left(C_{i} \mid s\right)$ for each concept $C_{i}$ using the n-ary classification mode of GBDT and normalizing the resulting scores. 0.2 is used as the importance threhold on the resulting scores. The training set is computed automatically: positive examples for each class $C$ are sampled from a pre-computed global cluster aligned with the target class 
Table 2: Features for lexicon element $e$ and concept $C$

\begin{tabular}{|c|c|}
\hline Description & Source \\
\hline capitalization ratio, string freq. & Web corpus \\
\hline relative freq. of $C$ in the set of tags for $e$ 's pages & Wikipedia \\
\hline $\begin{array}{l}\text { distrib. sim. with centroid of aligned cluster CL } \\
\% \text { of centroid features shared with } e \\
\text { distrib. sim. with centroid of S (concept seed set) } \\
\% \text { of centroid features shared with } e, \text { etc. }\end{array}$ & Web corpus \\
\hline $\begin{array}{l}\% \text { of high PMI query/doc. contexts for } \mathrm{S} \\
\text { which cooccur with } e \text {, etc. }\end{array}$ & $\begin{array}{l}\text { query log, } \\
\text { clicked docs }\end{array}$ \\
\hline
\end{tabular}

Table 3: Set of experimental lexicons

\begin{tabular}{|l|l|l|l|l|l|}
\hline Lexicon & Actor & Manufacturer & Movie & $\begin{array}{l}\text { TV } \\
\text { Show }\end{array}$ & $\begin{array}{l}\text { Magazine } \\
\text { Newspaper }\end{array}$ \\
\hline Size & 376,653 & 51596 & 120961 & 14012 & 5806 \\
\hline \hline
\end{tabular}

Table 4: Detecting concept label importance (F-1 measure)

\begin{tabular}{|l|l|l|l|l|l|}
\hline Method & Actor & Man. & Movie & TVShow & Mag. \\
$B_{i}$ & 0.46 & 0.66 & 0.51 & 0.86 & 0.60 \\
\hline$I_{C M}$ & $\mathbf{0 . 9 1} \dagger$ & $\mathbf{0 . 8 7} \dagger$ & $0.74 \dagger$ & $\mathbf{0 . 8 7}$ & $\mathbf{0 . 8 8} \dagger$ \\
\hline$I_{M M}$ & $0.84 \dagger$ & 0.60 & $\mathbf{0 . 8 7} \dagger$ & 0.61 & $0.78 \dagger$ \\
\hline \hline
\end{tabular}

(see Feature Set discussion below).

Feature Set In addition to string ambiguity clues and Wikipedia category information, we derive a large set of data source-specific concept representations and features from a large 2008 Web crawl, 1 year worth of query logs and 3 months' worth of queries together with clicked Web pages (examples in Table 2). Local concept representations are a) the centroid of a concept seed set $S$ or b) the top 100 sentence, query or document contexts ranked by average PMI (Pointwise Mutual Information) with the elements of S. Global representations are 2597 semantic clusters computed using the Cluster-by-Committee algorithm [2] on 1/5th of the 2008 Web crawl and automatically correlated with the lexicons and concepts of interest based on the relative overlap in the element set or centroid feature set.

\section{Detecting Label Importance: Evaluation}

For each of the 5 lexicons in Table 3 , we randomly sample 400 examples from the intersection of each semantic lexicon with a set of frequent queries and annotate them as described above. We compare $I_{C M}$ and $I_{M M}$ with a baseline $B_{i}$ which assumes that the target label is always important for a given lexicon string (a 10-fold cross validation setup is used). $I_{M M}$ uses a separate (automatically constructed) training set, but is evaluated on the same test set subsets as $I_{C M}$. The results in Table 4 show that concept label importance can be reliably detected across lexicons, especially by $I_{C M}$ (improvements over baseline are statistically significant at the 0.95 level). Errors are due to the lexical diversity of media lexicons (e.g., "omen", "gandhi", "night" are all Movies), noisy global clusters (lauren london in the Musician cluster), etc.

\subsection{QIS with Enhanced Lexicons}

Given the 5 target lexicons, we experiment with the following lexicon modification types:

1. Lexicon Filtering $f\left(I_{C M}\right)$ and $f\left(I_{M M}\right)$ denote two filtering (string removal) operations based on the concept label importance information computed by $I_{C M}$ and $I_{M M}$. $I_{M M}$ is also used to derive a dominance filter $f\left(D_{M M}\right)$, which eliminates a string $s$ from lexicon $L$ for concept $C$ is $C$ is not the most likely label for $s$.

\begin{tabular}{|l|l|l|l|l|l|l|}
\hline $\begin{array}{l}\text { KB } \\
\text { Version }\end{array}$ & $\begin{array}{l}\mathbf{P} \text { @en } \\
\text { gen } \\
\text { aff }\end{array}$ & $\begin{array}{l}\text { Cov } \\
\text { gen } \\
\text { aff }\end{array}$ & $\begin{array}{l}\mathbf{P} \text { @ig } \\
\text { siff }\end{array}$ & $\begin{array}{l}\text { Cov } \\
\text { sig } \\
\text { aff }\end{array}$ & $\begin{array}{l}\mathbf{P} \text { @est } \\
\text { set }\end{array}$ & $\begin{array}{l}\text { Cov } \\
\text { test } \\
\text { set }\end{array}$ \\
\hline original & 74.7 & 546 & 69.1 & 307 & 81.5 & 1027 \\
\hline$a\left(I_{C M}\right)$ & $81.1 \dagger$ & 546 & $78 \dagger$ & 307 & $84.8 \dagger$ & 1027 \\
\hline$a\left(I_{M M}\right)$ & $80.3 \dagger$ & 546 & $75.1 \dagger$ & 307 & 82.9 & 1027 \\
\hline$f\left(I_{C M}\right)$ & $\mathbf{8 7 . 8} \dagger$ & 288 & $\mathbf{8 9 . 7} \dagger$ & 247 & $88.4 \dagger$ & 1038 \\
\hline$f\left(I_{M M}\right)$ & $84.8 \dagger$ & 302 & $87.2 \dagger$ & 253 & $86.2 \dagger$ & 1034 \\
\hline$f\left(D_{M M}\right)$ & $86.7 \dagger$ & 271 & $88.3 \dagger$ & 234 & $\mathbf{8 9 . 3} \dagger$ & 1035 \\
\hline
\end{tabular}

Table 5: Positive Impact of Semantic Enhancements on Lexicon Use in Query Interpretation

2. Lexicon Augmentation $a\left(I_{C M}\right)$ and $a\left(I_{M M}\right)$ denote feature additions based on non-binary label importance scores computed by the regression versions of $I_{C M}$ and $I_{M M}$ : given an interpretation $I$ of a query $q$ which contains tokens $t_{1}, \ldots t_{k}$ of type $C$, we use the importance scores $i m p\left(t_{i}, C\right)$ to derive interpretation-level features: e.g., $f(I)_{\text {Actor }(\text { avg })}=$ $\sum_{1}^{k}$ imp $\left(\right.$ Actor,$\left.t_{i}\right) / k$.

The research version of QIS is trained on 27,000 queries and separately tested on 2700 queries. Evaluating performance amounts to evaluating the ranking of query interpretations; the gold standard is represented by manually judged interpretations for a randomly selected set of queries. Relevant queries have at least 2 interpretations, at least one of which is judged as Excellent or Good in the manually labeled data. Test set queries are relevant queries for which interpretations have been generated based on the comprehensive set of QIS lexicons. Generally affected queries are relevant queries with at least 1 interpretation containing a semantic tag which matches a target concept; for Significantly affected queries, this tag belongs to a Good or Excellent interpretation.

Metrics and Discussion Precision@1 is the precision for the top ranked interpretation; Coverage is the number of relevant queries for which interpretations are generated. As seen in Table 5, adding features based on label importance information leads to an increase in precision on affected queries of $6.4 \%$ and an overall precision increase of $3.3 \%$ (both significant at the 0.95 level). Lexicon filtering based on label importance leads to significant precision increases: $13 \%$ on affected queries and $7 \%$ overall, but at the cost of a $23 \%$ reduction in queries significantly affected and $48 \%$ in the number of queries generally affected.

Related Work [3] extracts lexicons from structured Web data and filters them for NER use in product queries, with a reported $25 \%$ increase in word-level tagging performance over a lexicon-free baseline. We show that augmenting lexicons is a promising alternative to filtering and report on query interpretation performance in generic Web search rather than word-level tagging performance in a particular domain.

\section{REFERENCES}

[1] J. H. Friedman. Greedy function approximation: A gradient boosting machine. The Annals of Statistics, 29(5):1189-1232, 2001.

[2] P. Pantel and D. Lin. Discovering word senses from text. In Proceedings of KDD, pages 613-619, 2002.

[3] Y. Wang, R. Hoffmann, X. Li, and J. Syzmanski. Semi-supervised learning of semantic classes for query understanding - from the Web and for the Web. In Proceedings of the CIKM, 2009. 